\title{
Frequency Domain Approach to Blind Source Separation in ECG Monitoring by Wearable System
}

\author{
M Milanesi ${ }^{1}$, N Vanello ${ }^{1}$, V Positano ${ }^{2}$, MF Santarelli ${ }^{2}$, \\ R Paradiso ${ }^{3}$, D De Rossi ${ }^{4}$, L Landini ${ }^{4}$ \\ ${ }^{1}$ Department of Electrical Systems and Automation, University of Pisa, Italy \\ ${ }^{2}$ CNR Institute of Clinical Physiology, Pisa, Italy \\ ${ }^{3}$ Milior SpA, Prato, Italy \\ ${ }^{4}$ Interdepartmental Research Center E Piaggio, University of Pisa, Italy
}

\begin{abstract}
In this paper we present a method for removing artifacts from biomedical signals acquired by wearable systems, taking advantage of multichannel data acquisition since both artifacts and signals of interest show common features in different channels. In order to take into account the effects of the different paths from the source signals to the sensors, we propose a method based on blind separation of convolutive mixtures: the observed data are seen as linear mixtures of filtered source signals where neither the source signals nor the convolution and mixing processes are known. The only hypothesis we make to recover the original sources is the statistical independence among them. The proposed method was applied on real ECG signals corrupted by motion artifacts with satisfactory results.
\end{abstract}

\section{Introduction}

Wearable systems are designed to monitor vital signs in real life environments. These systems are based on flexible and smart technology: fabric electrodes realized with metal based yarns enable the realization of wearable and wireless instrumented garments capable of recording physiological signals and to be used by a subject during everyday activity. Monitored vital signs include breathing pattern, electrocardiogram (ECG), electromyogram (EMG), activity sensors.

Some undesired signals may be superimposed on vital signs of interest and must be considered artifacts. These artifacts may have a biological origin, like reciprocal contaminations of muscle activity and heart cycles respectively in ECG and EMG recordings. Other artifacts are typical of wearable systems employment and can be caused by displacements of the electrodes integrated in the textile garment during subject movements. These motion artifacts produce base line drifts which can compromise vital signs parameters extrapolation. In the ECG recordings it is possible that all these artifacts cause the loss of the main features, like QRS complex, Pand T waves.

Linear and non linear filtering [1] and adaptive filtering [2] can be listed among the techniques proposed in order to recover the features of interest from ECG recordings. Other approaches take advantage from the multichannel nature of the acquisitions with the aim of searching for common features present in different channels acquired simultaneously. These approaches may be useful if the frequency content of signal and noise overlap and filtering operation cannot reduce noise without loosing signal information. In blind source separation (BSS) methods the electrodes are assumed to measure a mixtures of some latent sources, and both the sources and the mixing process are unknown. Independent component analysis (ICA) [3] is a technique employed to solve BSS problem that starts the research of the sources from the hypothesis of statistical independence among them. This assumption implies that components belonging to different physiological phenomena can be extracted from the signals detected by the sensors.

In the basic or instantaneous ICA model no time delay is involved in the mixing process and the signals picked up by the electrodes are a linear mixture of the sources. Several applications of basic ICA in removing artifacts from biomedical signals have been presented [H1] [4]. However, if we want to account for different paths of the source signals to the electrodes or for spatio-temporal dynamics, as Anemuller hypothesize for neural processes in EEG recordings [5], we must introduce convolution in the mixing process and consider that the sources have different time delays in each observed signals. For this reason we model our acquisitions as a convolutive mixture of unknown sources.

In this paper we present a frequency domain approach $[5,6]$ to solve the convolutive mixtures problem 
exploiting the algorithms developed for the instantaneous ICA model since a convolution in time domain is a product in the frequency domain. We show how the proposed method can remove artifacts from ECG signals acquired by the wearable system developed by Smartex S.r.1 [7, 8], partner in MyHeart IST-2002-507816 project and Wealthy IST-2001-37778 project. The processed signals are compared with the same ECG leads acquired simultaneously with standard red dot electrodes.

\section{Methods}

The basic or instantaneous ICA model assumes that a set of measurements $x_{i}(t)$ are originated by a linear mixing process of some latent sources $s_{i}(t)$ :

$$
x_{i}(t)=a_{i 1} s_{1}(t)+\ldots+a_{i j} s_{j}(t)+\ldots+a_{i n} s_{n}(t)
$$

with $i=1,2, \ldots m, j=1,2, \ldots, n$ and $t=1,2, \ldots, T$ as we operate with discrete time signals. If we use a vector representation of $\boldsymbol{x}(t)=\left[x_{l}(t), \ldots, x_{m}(t)\right]^{T} \quad$ and $s(t)=\left[s_{1}(t), \ldots, s_{n}(t)\right]^{T}$, we can express equation (1) in matrix notation $\boldsymbol{x}(t)=\boldsymbol{A s}(t)$ where $\boldsymbol{A}$ is called the mixing matrix. Both the sources $s_{i}(t)$ and the mixing process, $\boldsymbol{A}$, are unknown. The hypothesis used in order to extract the original sources is that they are statistically independent. The goal is to estimate a matrix $\boldsymbol{W}$ called the unmixing matrix, such that $\boldsymbol{y}(t)=\boldsymbol{W s}(t)$ is an estimate of the original sources $s(t)$. In the following we assume the number of sources equals the number of acquired signals, thus $n=m$.

To account for the different paths of the latent sources to the electrodes we have to consider the filtering effect of the tissue interposed. Hence we have to introduce convolution in the mixing process:

$$
x_{i}(t)=\sum_{j=1}^{n} \sum_{k=1}^{L} a_{i j}(k) s_{j}(t-k) \text { for } i=1, \ldots, n
$$

where the elements of the mixing matrix $\boldsymbol{A}$ become finite impulse response (FIR) filters of length $L$.

Fourier transform techniques are useful when dealing with convolutive mixtures, because convolution operator becomes a product in the frequency domain $[5,6]$. Thus a short time Fourier transform (STFT) of the observed signals is performed and the one-dimensional sequence $x_{i}(t)$, which is a function of a single variable $t$, is converted into a two-dimensional function $X_{i}(f, t)$ :

$$
X_{i}(f, t)=\sum_{j=1}^{n} A_{i j}(f) S_{j}(f, t)
$$

where $S_{j}(f, t)$ is the time-frequency representation of the $i$ th source and $A_{i j}(f)$ is the Fourier transform of the elements of the mixing matrix $\boldsymbol{A}$. The frequency content of each $X_{i}(f, t)$ can be simplified by dividing the values of $f$ in a certain number of frequency bin (or intervals). The instantaneous ICA model is applied separately in each frequency bin and the independent components $\boldsymbol{Y}(f, t)=\boldsymbol{W}(f) \boldsymbol{X}(f, t)$ are estimate where $\boldsymbol{W}(f)$ is the unmixing matrix for the $\operatorname{bin} f$.

Several approaches have been proposed in order to solve the instantaneous ICA model. In this work we make use of the fast fixed point algorithm (FastICA) derived by Hyvarinen for maximization of nongaussianity evaluated by the Negentropy of estimated components [3].

As we are dealing with complex numbers the instantaneous algorithm must be modified, thus the one unit learning rule of the FastICA becomes:

$$
\mathbf{w}_{i} \leftarrow E\left\{\mathbf{x} f\left(\mathbf{w}_{i}^{H} \mathbf{x}\right)\right\}-E\left\{f^{\prime}\left(\mathbf{w}_{i}^{H} \mathbf{x}\right)\right\} \mathbf{w}
$$

where the transposition operator is changed with the herimian operator ()$^{H}$ and $E\{$.$\} stands for the expectation$ operator. The non linearity $f(\boldsymbol{y})$, which takes into account higher order cumulants to approximate the Negentropy of the data, converts from $f(\boldsymbol{y})=\tanh (\boldsymbol{y})$ to $f(\boldsymbol{y})=\tanh (\operatorname{Re}\{\boldsymbol{y}\})+\tanh (\operatorname{Im}\{\boldsymbol{y}\}) i[6]$.

As pre-processing steps, before performing ICA on $X_{i}(f, t)$, both a removal of the mean value and a whitening operation using PCA is performed: this operation simplifies the estimation of the unmixing matrix $W$ that becomes orthogonal with only $n(n-1) / 2$ degrees of freedom instead of $n^{2}$.

A frequency domain ICA approach, as the basic ICA, suffers from some ambiguities: the first ambiguity is that it is not possible to determine the order of the extracted independent components while, the second ambiguity, regards the fact that the independent components are always extracted up to a scale and a phase factor. Both these ambiguities affect the ICA model applied at each frequency bin. In order to solve the first ambiguity we need some method for choosing which components in different bins belong together and can be associated to the same source signal. Inter-frequency correlations of signals can be used [9], since two spectral envelopes belonging to the same source should have a higher correlation coefficient than the one they would have if they belonged to different sources. For this reason we compute the correlation coefficient between each $\left|Y_{i}\left(f_{k}, t\right)\right|$ extracted in one bin and the others $\left|Y_{j}\left(f_{k-1}, t\right)\right|$, with $j=1, \ldots, n$, belonging to the preceding bin. An iterative procedure looks for the highest correlation value and aligns the two components that have obtained it. These two components are eliminated from successive steps and the same practice is reiterated for the remaining $n-1$ components. This procedure is repeated until the last 
frequency bin is aligned with the preceding one.

To solve the second ambiguity the estimated components can be returned to the space of the observation by:

$$
X_{i} Y_{j}(f, t)=\left(W_{f}^{-1}\right)_{i j}\left(\begin{array}{c}
0 \\
\vdots \\
Y_{j}(f, t) \\
0
\end{array}\right)
$$

where $X_{i} Y_{j}(f, t)$ represents the $j$-th estimated independent component contribute in the $i$-th channel for the frequency $\operatorname{bin} f$, and $\left(\boldsymbol{W}_{f}^{-1}\right)_{i j}$ is the $i j$-th element of $\boldsymbol{W}_{f}^{-l}$.

After performing all this linear transformations, we can group the $X_{i} Y_{j}(f, t)$ in the following way:

$$
X_{i}^{\prime}(f, t)=\sum_{j} X_{i} Y_{j}(f, t)
$$

If some independent components, returned to the observation space, are not significant for the information provided by the channel $i$, they can be set to zero in (6). This procedure guarantees artifacts removal from a multichannel acquisition.

As we want to monitor the ECG signals, the related component extracted from convolutive mixtures must be identified automatically. We propose to exploit the periodicity of the ECG signal. If a short observation window (several seconds long) is chosen, the same periodicity can be found in the magnitude spectrogram [10]. This periodic pattern can be used to discriminate the ECG (or at least the QRS) component from other non periodic components. Signals with important periodic components show different mean value of the magnitude spectrogram autocorrelation function, from the one computed for non periodic signals. In fact, for the latter class of signals, the time over which a certain pattern is correlated is very short and settles rapidly to zero. An ECG related component can be identified fixing a proper threshold of the area of the autocorrelation function.

An inverse short time Fourier transform (ISTFT) can be used as final step to obtain the reconstructed signals in the time domain $x_{i}{ }^{\prime}(t)$ without artifacts components.

\section{Results}

The proposed method was tested on real ECG signals acquired by Smartex s.r.l. wearable system. The subject was asked to twist his bust in order to produce signals affected by artifacts.

Two precordial leads, V2 and V5 were registered with the wearable systems and with red dot electrodes placed just below the metal yarn ones. Red dot electrodes are considered the gold standards in this study. The analog filter was a band pass filter with cut off frequencies 0.3 $100 \mathrm{~Hz}$ followed by a notch filter at $50 \mathrm{~Hz}$. The sampling rate was $f_{s}=250 H z$ (sampling period $\left.T s=1 / f s=4 m s\right)$. Eight seconds long recordings were processed with convolutive ICA. The time-frequency representation of the data was obtained by a $\Delta t=120 \mathrm{~ms}$ Hamming window $\left(N=\Delta * T_{c}=3 \mathrm{C}\right.$ points) and the overlap between neighbouring windows was fixed at $90 \%$. The frequency domain ICA was carried out for the frequency bins included between $f_{l}=0 \mathrm{~Hz}$ and $f_{2}=50 \mathrm{~Hz}$, assuming that the main frequency ECG content is located in this interval. For the remaining bins no analysis was carried out and both the channels were left unchanged. Hyvarinen's FastICA algorithm (4) was employed for independent component research. The number of iterations for each algorithm's run was fixed at 50. After two independent components had been estimated in each bin and aligned according to correlation coefficient technique, they were returned to the observation space by (5). Then, concentrating the analysis in the first ECG channel, the most periodic magnitude spectrogram among the two components were searched employing the autocorrelation function method described in the previous section The most periodic component was associated to the ECG source, while the other was considered an artifact component and set to zero in (6). A inverse STFT was applied to obtain time domain evolution of the signals $x_{i}{ }^{\prime}(t)$, where $i=1$ indicates the V2 while $i=2$ indicates the V5 precordial lead.

To evaluate the performance of the proposed method, the original wearable system's acquisitions $x_{i}(t)$ and the algorithm output signals $x_{i}{ }^{\prime}(t)$ were compared with the same ECG lead signals $x_{r i}(t)$ recorded with standard red dot electrodes (their time evolution is showed in figure 1).
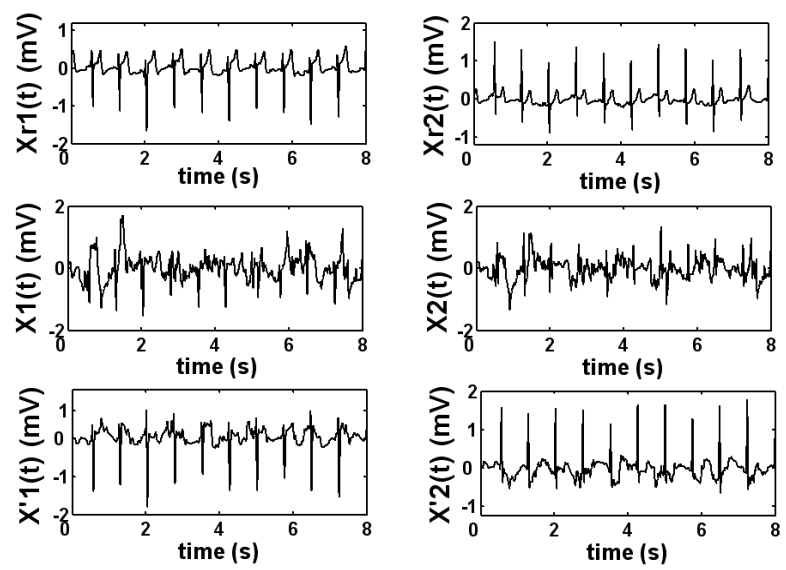

Figure 1. Time evolution of acquired signals $x_{i}(t)$ and $x_{r i}(t)$ and processed signals $x_{i}^{\prime}(t)$. 
Hence the correlation coefficients between the magnitude spectrograms of $x_{i}(t)$ and $x_{r i}(t)$ and between $x_{i}^{\prime}(t)$ and $x_{r i}(t)$ were estimated. These correlation coefficients were used as quality indexes to asses the algorithm performance. The spectrograms were obtained by a STFT using the same window specifics employed for the time frequency representation of the original wearable system data. The correlation coefficients were evaluated in every frequency bin inside the $f_{1}-f_{2}$ interval $(0-50 \mathrm{~Hz})$, where we carried out the independent components research. The total number of frequency bins analyzed is 7, given a frequency resolution of $\Delta f=1 /\left(N * T_{S}\right)=8.333 \mathrm{~Hz}$. In figure 2 the correlation coefficients between magnitude spectrograms as function of the frequency bins are shown.
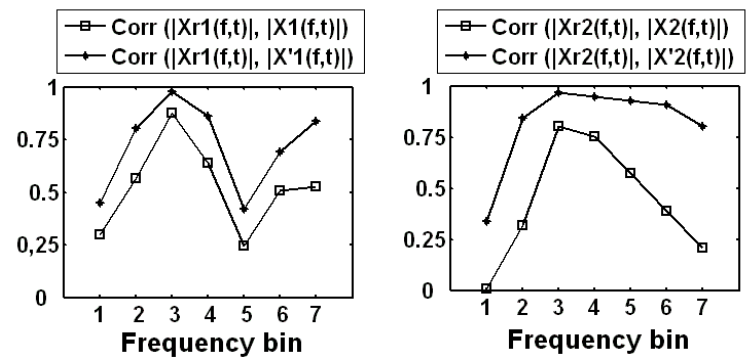

Figure 2. Correlation coefficients between magnitude spectrograms $\operatorname{Xri}(f, t)$ and $X(i f, t)$ and between $\operatorname{Xri}(f, t)$ and $X^{\prime} i(f, t)$. as function of frequency bins.

\section{Discussion and conclusions}

In this paper we have proposed a method for monitoring ECG signals by a frequency domain approach to blind source separations. Starting from the multichannel nature of the acquisitions we have modelled our signals as a convolutive mixture of independent components. The ECG and artifacts components were separated from multichannel acquisitions in frequency domain, where we could exploit the algorithms derived for instantaneous ICA model.

Two ECG precordial leads were acquired by Smartex s.r.1 wearable systems and processed with our algorithm. The original data and the results achieved by the proposed method were compared with the same ECG leads signals acquired with standard red dot electrodes. The correlation coefficients comparison in figure 2 indicates that the application of our algorithm to wearable systems recordings produces ECG signals more similar to the standard red dot ones than the original acquisitions. The convolutive ICA technique, as presented in this paper, can thus be employed to improve the features of ECG signals acquired by wearable systems affected by artifacts.
A frequency domain approach to convolutive ICA allows to carry out the independent component research only in those bins where the artifacts and the desired signals overlap. This method can be extended to a wide collection of noisy multichannel acquisitions as it is completely blind and doesn't makes any hypothesis on the signals features.

\section{Acknowledgements}

This work was supported by E. U. project MyHeartIST-2002-507816.

\section{References}

[1] Eugene NB. Biomedical signal processing and signal modeling, 2001; J. Wiley-Interscience Ed.

[2] Almebar V, Albiol A. A new adaptive scheme for ECG enhancement Signal Process. 1999; 75: 253-263

[3] Hyvarinen A, Karhunen J, Oja E. Independent component analysis 2001; J. Wiley \& Sons Ed.

[4] James CJ, Hesse CW. Independent Component Analysis for biomedical signals. Physiol. Meas. 2005; 26: 15-39.

[5] Anemuller J, Sejnowski TJ, Makeig S. Complex spectraldomain independent component analysis of electroencephalographic data. Neural Netwoks 2003; 16, 1311-1323.

[6] Smaragdis P. Blind separation of convolved mixtures in the frequency domain. Neurocomputing 1998; 22: 21-31.

[7] Paradiso R. Tessuto in maglia per il monitoraggio di segnali vitali. 2003: Italian Patent N. FI2003A000308.

[8] Paradiso R, Loriga G, Taccini N. A Wearable Health Care System Based on Knitted Integrated Sensors, IEEE Trans. Inf. Technol. Biomed., in printing.

[9] Sawada H, Mukai R, Araki S, Makino S. A Robust and Precise Method for Solving the Permutation Problem of Frequency-Domain Blind Source Separation, IEEE Trans. Speech Audio Processing 2004; 12: 530-538.

[10] Milanesi M, Vanello N, Positano V, Santarelli MF, De Rossi D, Landini L. An Automatic Method for Separation and Identification of Biomedical Signal from Convolutive Mixtures by Independent Component Analysis in the Frequency Domain. 2005; Proc. of the $5^{\text {th }}$ WSEAS Int. Conf. on SSIP, Corfù, Greece, 74-79.

Address for correspondence

Dr. Matteo Milanesi

Dep. of Electr. Systems and Automat, Fac. of Engineering, Via Diotisalvi 2, 56126, Pisa, Italy matteo.milanesi@ing.unipi.it 